\title{
PERCEPÇÃO DO SUPORTE FAMILIAR EM IDOSOS DE BAIXA RENDA E FATORES ASSOCIADOS*
}

\author{
Luciana Araújo dos Reis ${ }^{1}$, Gilson de Vasconcelos Torres", Thaiza Teixeira Xavier ${ }^{3}$, Richardson Augusto \\ Rosendo da Silva ${ }^{4}$, Isabelle Katherinne Fernandes Costa ${ }^{5}$, Felismina Rosa Parreira Mendes ${ }^{6}$
}

\footnotetext{
${ }^{1}$ Doutoranda em Ciências da Saúde pelo Programa de Pós-Graduação em Ciências da Saúde da Universidade Federal do Rio Grande do Norte (UFRN). Docente da Universidade Estadual do Sudoeste da Bahia. Bahia, Brasil. E-mail: cianareis@hotmail.com

${ }^{2}$ Doutor em Enfermagem. Docente do Programa de Pós-Graduação em Ciências da Saúde da UFRN. Rio Grande do Norte, Brasil. E-mail: gvt@ufrnet.br

${ }^{3}$ Doutora em Ciências da Saúde. Docente do Departamento de Fisioterapia. UFRN. Rio Grande do Norte, Brasil. E-mail: thaizax@yahoo.com.br

${ }^{4}$ Doutor em Ciências da Saúde. Docente do Departamento de Enfermagem da UFRN. Rio Grande do Norte, Brasil. E-mail: rirosendo@yahoo.com.br

${ }^{5}$ Doutoranda do Programa de Pós-Graduação em Enfermagem da UFRN. Rio Grande do Norte, Brasil. E-mail: isabellekfc@ yahoo.com.br

${ }^{6}$ Doutora em Sociologia. Professora da Universidade de Évora. Portugal. E-mail: fm@uevora.pt
}

RESUMO: Este estudo teve por objetivo identificar a percepção do suporte familiar em idosos de baixa renda e os fatores associados. Trata-se de estudo transversal, com amostra de 235 idosos, residentes no município de Jequié-BA. O instrumento foi constituído por: dados sociodemográficos e de saúde; Mini-exame do Estado Mental; Índice de Barthel e Inventário de Percepção do Suporte Familiar. A análise estatística foi realizada com nível de significância $p<0,05$, mediante realização do teste do Qui-Quadrado. Verificou-se diferença estatística significativa entre as médias do domínio Afetividade-consistência e as variáveis presença de sequelas $(p=0,042)$ e comprometimento das atividades básicas de vida diária $(p=0,033)$, entre as médias do domínio Adaptação-familiar e nível de escolaridade analfabetos $(p=0,012)$, e entre o domínio Autonomia e as variáveis estado civil casado $(p=0,013)$, realização de tratamento $(p=0,032)$ e comprometimento das atividades básicas de vida diária $(\mathrm{p}=0,011)$. Desta forma evidencia-se que a maioria dos idosos avaliados apresentou comprometimento da percepção do suporte familiar.

DESCRITORES: Saúde do idoso. Atividades cotidianas. Fatores socioeconômicos. Família.

\section{PERCEPTION OF FAMILY SUPPORT AMONG LOW-INCOME ELDERLY AND ASSOCIATED FACTORS}

\begin{abstract}
This study aims to identify the perception of family support among low-income elderly and associated factors. It is crosssectional study with a sample of 235 elderly residents of Jequié, Bahia, Brazil. The instrument consisted of: demographics and health; Mini-Mental State Examination, Barthel Index, and Perceived Family Support Inventory. Statistical analysis was performed with a $p<0.05$ level of significance, upon completion of the Chi-Square. There was a statistically significant difference between the averages for Consistency-affection domain and presence of consequences variables $(p=0.042)$ and impairment of basic daily living activities $(p=0.033)$; and between Family-adaptation domain averages and illiterate education levels ( $p=0.012)$; and between the Autonomy domain and married state variables $(p=0.013)$, completion of treatment $(p=0.032)$, and impairment of basic daily living activities $(p=0.011)$. Thus, it is evident that most seniors tested showed impaired perception of family support.
\end{abstract}

DESCRIPTORS: Elderly health. Daily activities. Socioeconomic factors. Family

\section{PERCEPCIÓN DEL APOYO FAMILIAR A ANCIANOS DE BAJOS INGRESOS Y FACTORES ASOCIADOS}

\begin{abstract}
RESUMEN: Este estudio tiene como objetivo identificar la percepción del apoyo familiar a ancianos de bajos ingresos y los factores asociados. Es un estudio transversal con una muestra de 235 ancianos residentes en Jequié, Bahia, Brasil. El instrumento consiste en: datos demográficos y de salud, Mini Examen del Estado Mental, índice de Barthel y el Inventario de Percepción de Apoyo a la Familia. $\mathrm{El}$ análisis estadístico se realizó con un nivel de significación $\mathrm{p}<0,05$, mediante prueba de Chi-cuadrado. Se verifican diferencias estadísticamente significativas entre las medias del dominio Afectividad-Consistencia y las variables de presencia de secuelas $(p=0,042)$ y discapacidad en las actividades de la vida diaria $(p=0,033)$, entre las medias del domino Adaptación-familiar y el nivel de escolarización analfabetos $(\mathrm{p}=0,012)$, y entre el dominio Autonomía y las variables estado civil casado $(\mathrm{p}=0,013)$, la finalización del tratamiento $(\mathrm{p}=0,032)$ y la discapacidad en las actividades básicas de la vida diaria $(\mathrm{p}=0,011)$. Por lo tanto, es evidente que la mayoría de los pacientes de edad avanzada mostraron alteración en la percepción del apoyo familiar.
\end{abstract}

DESCRIPTORES: Salud del anciano. Actividades cotidianas. Factores socioeconómicos. Familia.

* $3^{\circ}$ lugar - Prêmio Maricel Manfredi. 


\section{INTRODUÇÃO}

Os idosos constituem um grupo de risco dentro das prioridades de saúde pública, pois são mais suscetíveis a doenças, acidentes, violência e sistema familiar vulnerável. Dessa forma, registra-se neles uma fragilização e probabilidade de agravos maiores e, em consequência, evidenciando-se como o grupo que, proporcionalmente, consome mais serviços de saúde. ${ }^{1}$

Dentro dessa perspectiva em que o idoso representa maior fragilização e risco aumentado de agravos à saúde, a questão da família se constitui em um importante fator a ser analisado. Países em desenvolvimento carecem de uma rede de suporte formal eficiente e a família acaba se tornando a responsável por auxiliá-los em quaisquer necessidades específicas que surjam, seja material ou emocional. O ambiente familiar, nesse sentido, constitui-se na mais importante rede de suporte que o idoso possui em nosso país. Independentemente do arranjo e da estrutura familiar (número de membros, papéis definidos entre os membros) do idoso, faz-se importante salientar que é fundamental a qualidade do suporte familiar dessas pessoas. ${ }^{2}$

O suporte familiar se mostra um construto de difícil operacionalização, sendo uma de suas definições como parte da rede informal e mais próxima de relacionamentos, na qual o indivíduo é beneficiado por meio do contato e das trocas mantidas com seus familiares, podendo desenvolver maior resiliência e bem-estar psicológico e sendo auxiliado na manutenção de respostas mais adequadas diante de eventos que tendem a comprometer a saúde. ${ }^{3}$ Algumas das funções do suporte familiar são: dar e receber informações, proporcionar auxílio material e emocional, dar às pessoas o senso de amor e valorização, possibilitar a construção e manutenção de uma identidade social, além do auxílio na atribuição de sentido às experiências de vida. ${ }^{4}$

O suporte familiar contribui de maneira significativa para a manutenção e a integridade física e psicológica do indivíduo. Seu efeito é tido como benéfico, no membro da família que o recebe, na medida em que o suporte é percebido como disponível e satisfatório. Desta forma, compreende-se ser fundamental para o planejamento assistencial adequado ao idoso, a compreensão de seu contexto familiar e seu cotidiano, o que implica na compreensão das questões que envolvem a formação e a dinâmica de funcionamento das famílias em geral, a identificação da presença de incapacidades funcionais e as suas principais necessidades de cuidado para lidar com as incapacidades. ${ }^{5-6}$

A família é um sistema dinâmico, em interação dialógica, que pretende (ou deve pretender) ajudar a pessoa a desenvolver uma presença afetiva, responsável e livre no mundo. Cada pessoa tem e terá, ao longo de sua existência, várias famílias (a de seus ancestrais, a de sua infância, a de sua adolescência, a de sua vida adulta e de sua velhice), assumindo em cada fase características peculiares, mas mantendo a função primordial, a de preservar a integridade física e emocional de seus membros e do próprio grupo, propiciando o seu desenvolvimento. ${ }^{7}$ Nesse estudo foram adotadas como família as pessoas que residem no mesmo ambiente familiar do idoso, participando assim de seu dia-a-dia.

O conhecimento das condições de apoio familiar, ambiente físico e psíquico de vida do idoso é importante para o desenvolvimento de mecanismos de assistência domiciliária à saúde do idoso, $\mathrm{e}$ impõe-se como indispensável, em todos os níveis sociais, seja por suas características de respeito à individualidade e privacidade do idoso, seja pela possibilidade de minimizar o estresse inerente à atenção à saúde, aumentando a possibilidade de aceitação dos cuidados e consequente eficiência dos procedimentos. ${ }^{8}$

Portanto, observa-se a necessidade cada vez mais premente de serem estabelecidos esquemas assistenciais mais efetivos e dinâmicos capazes de assistir as demandas crescentes dos idosos e de suas famílias, de forma a permitir que ambos encontrem conjuntamente uma solução terapêutica mais efetiva e adequada, em que o equilíbrio familiar seja a meta, melhorando assim a assistência ao idoso e diminuindo os custos emocionais da própria família, favorecendo a manutenção da capacidade funcional e melhoria na qualidade de vida destes. Nesta perspectiva, este estudo tem por objetivo identificar a percepção do suporte familiar em idosos de baixa renda e os fatores associados.

\section{MÉTODO}

Trata-se de uma pesquisa do tipo analítica, com delineamento transversal, realizada no município de Jequié, localizado no interior da Bahia, Região Nordeste do Brasil, no período de setembro de 2008 a janeiro de 2009. A população total do estudo foi representada por 1506 idosos, sendo a amostra constituída por 235 idosos, selecionada de forma aleatória simples, através de sorteio com 
reposição, em Unidades de Saúde do Bairro do Jequiezinho. O valor da amostra foi calculado com base nos valores da média $(12,65)$, desvio-padrão $( \pm 3,61)$ e margem de erro de $5 \%$, encontrados nos domínios adaptação familiar e autonomia do Inventário de Percepção do Suporte Familiar no Teste Piloto, realizado com 36 idosos participantes de um Grupo de Convivência para a terceira idade.

Os critérios de inclusão no estudo foram apresentar condições mentais (pontuação acima de 23 pontos no Mini-exame do Estado Mental) $(\mathrm{MEEM})^{9}$ para responder ao instrumento da pesquisa e concordar em participar da pesquisa ou ter sua participação autorizada pelo cuidador, firmando o Termo de Consentimento Livre e Esclarecido.

O instrumento de coleta de dados foi constituído de quatro partes. A primeira parte, representada pela caracterização sociodemográfica: idade, sexo, escolaridade, valor da renda, estado civil; e condições de saúde (presença ou ausência de problemas de saúde e de sequelas). A segunda parte, referente ao $\mathrm{MEEM}^{9}$, para avaliar o estado cognitivo do idoso (pontuação acima de 23 pontos). A terceira parte foi composta pelo Índice de Barthel, utilizado para avaliar a capacidade funcional, e a quarta parte, composta pelo Inventário de Percepção de Suporte Familiar, para avaliar o ambiente familiar quanto à adaptação, autonomia e afetividades.

O Índice de Barthel é utilizado para avaliar capacidade funcional, sendo composto por 10 atividades básicas de vida diária: alimentação, banho, higiene pessoal, vestir-se, intestinos, bexiga, transferência para higiene intíma, transferência - cadeira e cama, deambulação e subir escadas. O escore correspondente à soma de todos os pontos obtidos, sendo considerado independente o indivíduo que atingir a pontuação total, isto é, 100 pontos. Pontuações até 75 pontos corresponde a dependência leve, até 50 pontos, dependência moderada, e abaixo de 25 , indicam dependência grave. ${ }^{10}$

O Inventário de Percepção de Suporte Familiar (IPSF) ${ }^{12}$ consiste em construir, validar e fornecer maneiras de mensurar o construto de suporte familiar. É composto por 42 itens, sendo dividido em três dimensões, sendo o primeiro fator denominado de Afetivo-Consistente. O segundo fator é denominado de Adaptação Familiar, que é pontuado de maneira invertida. Para efeitos de correção e análise, este fator tem pontuação invertida, sendo que quando pontuado zero, vale dois; um, permanece igual; e dois, vale zero. A última dimensão é a Autonomia.
As questões são do tipo likert de três pontos, nas quais o respondente assinala nunca (0), quase nunca (1) ou quase sempre (2).

No domínio Afetividade-Consistência quanto maior a pontuação obtida, pior é a percepção do suporte familiar e a pontuação obtida é assim classificada: baixo (0-21 pontos), médio-baixo (2228 pontos), médio-alto (29-33 pontos), e alto (34-42 pontos). No domínio Adaptação-familiar, quanto maior a pontuação obtida, melhor é o suporte familiar percebido, sendo a pontuação classificada em: baixo (0-18 pontos), médio-baixo (19-21 pontos), médio-alto (22-23 pontos), alto (24-26 pontos).

No domínio Autonomia, terceiro fator, quanto maior a pontuação obtida pior é a percepção do suporte familiar e a pontuação obtida é classificada: Baixo (0-9 pontos), Médio-Baixo (10-12 pontos), Médio-Alto (13-14 pontos), Alto (15-16 pontos).

Para aplicação do teste Estatístico do Qui-quadrado utilizou-se os valores encontrados na mediana da pontuação de cada fator, ficando a distribuição da seguinte forma:

- Autonomia: bom $(\leq 14)$ e ruim $(>14)$ pontos;

- Afetividade-Consistência: boa $(<38)$ e ruim ( $\geq 38$ ) pontos;

- Adaptação-Familiar: bom ( $\geq 21)$ e ruim $(<21)$ pontos.

Os dados coletados foram organizados em banco de dados eletrônicos, por meio de digitação em planilha do Programa Estatístico SPSS, versão 13.0. Na análise descritiva foi feita a distribuição de frequências absolutas, a distribuição percentual, e média e desvio padrão ( \pm ). Na abordagem analítica foi realizado o teste Qui-Quadrado, considerando-se um nível de significância $\mathrm{p}<0,05$.

Esta pesquisa seguiu os princípios éticos presentes na declaração de Helsinki e da Resolução ${ }^{\circ}$ 196/96 do Conselho Nacional de Saúde. Os protocolos de pesquisa foram avaliados e aprovados pelo Comitê de Ética em Pesquisa de Seres Humanos da Universidade Estadual do Sudoeste da Bahia (Parecer $n^{\circ}$ 189/2008).

\section{RESULTADOS}

A amostra do estudo constituiu-se de 235 idosos, com média de idade de 73,52 $( \pm 9,41)$ anos, sendo $69,80 \%(\mathrm{n}=164)$ do sexo feminino, casados $45,50 \%$ (n=107) e com renda familiar de um salário mínino $(79,10 \%)(\mathrm{n}=186)$.

Em relação às condições de saúde, 93,20\% $(n=219)$ dos idosos apresentavam problemas de 
saúde, sendo as patologias do aparelho osteomuscular as mais predominantes. A presença de sequelas esteve presente em apenas $23,00 \%(n=54)$ dos idosos, sendo mais frequentes as sequelas neurológicas $(15,70 \%)(\mathrm{n}=37)$. O uso de medicação controlada foi relatado por $76,60 \%(n=180)$ da amostra, sendo mais comum o uso de duas vezes ao dia $(37,00 \%)(n=87)$.

Com relação às atividades básicas de vida diária, $69,80 \%(\mathrm{n}=164)$ dos idosos foram classificados como dependentes, sendo mais prevalentes os idosos com dependência do tipo leve $(41,70 \%)$ $(\mathrm{n}=98)$. A pontuação obtida no Índice de Barthel oscilou entre 0 a 100 pontos, com média de 71,19 $( \pm 28,46)$ pontos, com mínimo de 10 pontos e máximo de 100 pontos.

Quanto à percepção do suporte familiar, no domínio Afetividade-Consistência, a maioria dos idosos apresentou pontuação alta $(71,10 \%)$ $(\mathrm{n}=167)$, com média de $33,54( \pm 11,74)$ pontos. No domínio Adaptação-familiar, houve uma maior frequência de idosos com pontuação baixa $(44,70)$ $(n=105)$, com média de 16,26 $( \pm 9,27)$ pontos. Enquanto que no domínio Autonomia houve uma maior distribuição de idosos com pontuação alta $(48,90)(n=115)$, com média de 13,91 $( \pm 3,04)$. Na pontuação total do IPSF houve uma maior frequência de idosos com pontuação alta $(40,90)$ $(\mathrm{n}=96)$ (Tabela 1).
Tabela 1 - Distribuição da pontuação dos idosos de baixa renda no IPSF. Jequié-BA, 2009

\begin{tabular}{lrr}
\hline Variável & $\mathbf{n}$ & \multicolumn{1}{c}{$\%$} \\
\hline Afetividade-Consistência & & \\
baixo (0-21 pontos) & 38 & 16,17 \\
médio-baixo (22-28 pontos) & 18 & 7,66 \\
médio-alto (29-33 pontos) & 12 & 5,11 \\
alto (34-42 pontos) & 167 & 71,06 \\
Adaptação Familiar & & \\
baixo (0-18 pontos) & 105 & 44,68 \\
médio-baixo (19-21 pontos) & 13 & 5,53 \\
médio-alto (22-23 pontos) & 19 & 8,09 \\
alto (24-26 pontos) & 98 & 41,7 \\
Autonomia & & \\
baixo (0-9 pontos) & 13 & 5,53 \\
médio-baixo (10-12 pontos) & 41 & 17,45 \\
médio-alto (13-14 pontos) & 66 & 28,09 \\
alto (15-16 pontos) & 115 & 48,94 \\
\hline Total & $\mathbf{2 3 5}$ & $\mathbf{1 0 0 , 0 0}$ \\
\hline
\end{tabular}

Com a aplicação do Teste do Qui-quadrado entre as médias do domínio Autonomia do Inventário de Percepção do Suporte Familiar e as variáveis do estudo verificou-se diferença estatística significativa com estado civil casado $(p=0,013)$, realização de tratamento $(p=0,032)$ e comprometimento das Atividades básicas de vida diária/ ABVDs ( $p=0,011)$ (Tabela 2).

Tabela 2 - Distribuição das médias do Domínio Autonomia, segundo estado civil, tratamento e ABVD. Jequié-BA, 2009.

\begin{tabular}{|c|c|c|c|c|c|c|c|}
\hline \multirow{2}{*}{ Autonomia } & \multicolumn{2}{|c|}{ Bom $(\leq 14)$} & \multicolumn{2}{|c|}{ Ruim (>14) } & \multicolumn{2}{|c|}{ Total } & \multirow{2}{*}{$p$-valor } \\
\hline & $\mathrm{n}$ & $\%$ & $\mathbf{n}$ & $\%$ & $\mathbf{n}$ & $\%$ & \\
\hline \multicolumn{8}{|l|}{ Estado Civil } \\
\hline Solteiro & 166 & 79,43 & 43 & 20,57 & 209 & 100 & \multirow{2}{*}{0,013} \\
\hline Casado & 15 & 57,69 & 11 & 42,31 & 26 & 100 & \\
\hline \multicolumn{8}{|l|}{ Tratamento } \\
\hline Sim & 144 & 73,85 & 51 & 26,15 & 195 & 100 & \multirow{2}{*}{0,032} \\
\hline Não & 37 & 92,5 & 3 & 7,5 & 40 & 100 & \\
\hline \multicolumn{8}{|l|}{ ABVD } \\
\hline Independente & 46 & 64,79 & 25 & 35,21 & 71 & 100 & \multirow{2}{*}{0,011} \\
\hline Dependente & 135 & 82,32 & 29 & 17,68 & 164 & 100 & \\
\hline
\end{tabular}

Nas médias do domínio Afetividade-Consistência observou-se diferença estatística significativa com presença de sequelas $(p=0,042)$ e comprometimento das ABVD $(\mathrm{p}=0,033)$ (Tabela 3$)$. 
Tabela 3 - Distribuição das médias do domínio Afetividade-Consistência, segundo presença de sequelas e ABVD. Jequié-BA, 2009.

\begin{tabular}{lccccccc}
\hline \multirow{2}{*}{ Afetividade-Consistência } & \multicolumn{2}{c}{ Boa (< 38) } & \multicolumn{2}{c}{ Ruim $(\geq \mathbf{3 8})$} & \multicolumn{2}{c}{ Total } & \\
\cline { 2 - 6 } & $\mathbf{n}$ & $\mathbf{\%}$ & $\mathbf{n}$ & $\mathbf{0}$ & $\mathbf{n}$ & $\mathbf{p}$ & p-valor \\
\hline Sequelas & & & & & & & \\
Sim & 29 & 53,70 & 25 & 46,30 & 54 & 100,00 & 0,042 \\
Não & 69 & 38,12 & 112 & 61,88 & 181 & 100,00 & \\
ABVD & & & & & & & \\
Independente & 37 & 52,11 & 34 & 47,89 & 71 & 100,00 & 0,033 \\
Dependente & 61 & 37,20 & 103 & 62,80 & 164 & 100,00 & 0,03 \\
\hline
\end{tabular}

E nas médias do domínio Adaptação-Familiar constatou-se diferença estatística significativa apenas com relação ao nível de escolaridade não alfabetizado $(\mathrm{p}=0,012)$ (Tabela 4).

Tabela 4 - Distribuição das médias do domínio Adaptação Familiar segundo escolaridade. JequiéBA, 2009.

\begin{tabular}{|c|c|c|c|c|c|c|c|}
\hline \multirow{2}{*}{ Escolaridade } & \multicolumn{2}{|c|}{ Bom $(\geq 21)$} & \multicolumn{2}{|c|}{ Ruim $(<21)$} & \multicolumn{2}{|c|}{ Total } & \multirow{2}{*}{$p$-valor } \\
\hline & n & $\%$ & $\mathbf{n}$ & $\%$ & $\mathrm{n}$ & $\%$ & \\
\hline Analfabeto & 80 & 57,97 & 58 & 42,03 & 138 & 100,00 & ? \\
\hline Alfabetizado & 40 & 41,24 & 57 & 58,76 & 97 & 100,00 & \\
\hline
\end{tabular}

\section{DISCUSSÃO}

A família constitui o principal sistema de suporte do idoso, apesar das dificuldades vivenciadas na contemporaneidade. Assim, nos casos de vulnerabilidade do sistema familiar do idoso e do sistema formal, ou de abandono do idoso por ambos, a principal consequência é a exclusão do idoso de sua coletividade, para "depositá-lo" num asilo, com sérios efeitos negativos sobre sua qualidade de vida. ${ }^{11}$

No contexto familiar, muitos são os sentimentos construídos na convivência entre o idoso e seus familiares. O afeto, a ajuda mútua e a compreensão são aspectos essenciais que devem existir no relacionamento idoso/família. Assim, o convívio se torna agradável e os idosos conseguem viver de forma harmoniosa junto a seus entes queridos. Por outro lado, em muitos casos, identifica-se que tal convivência apresenta turbulências, podendo levar a desentendimentos e desgastes no relacionamento. Isso pode acontecer por diversos motivos, seja por divergência de ideias ou devido à dependência do idoso em relação a seus íntimos. ${ }^{12}$

Os idosos do presente estudo segundo o Inventário de Percepção do Suporte Familiar, apresentaram comprometimento na percepção do suporte familiar nos três domínios Afetividade-Consistência, Adaptação-Familiar e Autonomia, demonstrando desta forma que a convivência familiar destes idosos não se apresenta adequada, o que pode interferir na qualidade de vida destes idosos.

O domínio Afetividade-Consistência envolve questões a respeito da expressão de afetividade entre os membros, seja verbal ou não, interesse, simpatia, acolhimento, consistência de comportamentos e habilidades na resolução de problemas. ${ }^{13}$ A elevada pontuação apresentada pela maioria dos idosos neste domínio demonstra que as famílias não estão preparadas para vivenciar emoções apropriadas frente a uma variedade de estímulos, tanto de bem-estar quanto de mal-estar. Desta forma, a falta de preparo da família acarreta prejuízos na qualidade do cuidado prestado ao idoso.

O domínio Adaptação Familiar questiona os sentimentos negativos em relação ao grupo familiar, como raiva, isolamento, exclusão, falta de compreensão entre outros, que apontam a ausência de adaptação no grupo. ${ }^{13}$ Os dados encontrados neste estudo revelam que a maioria das famílias não possui habilidade de transformar a estrutura de poder e as regras de relacionamentos em resposta a situações de estresse.

No domínio Autonomia são avaliadas as relações de confiança, liberdade e privacidade entre os membros. ${ }^{13}$ Diante dos dados encontrados nesse estudo, fica evidenciado que os idosos entrevistados 
não possuem incentivo e liberdade para se expressar e ter autonomia no ambiente familiar. A perda da autonomia para o idoso representa uma morte social que anuncia a morte física do indivíduo e com a qual a sociedade moderna, secularizada e racional, tem enormes dificuldades de lidar.

Com a aplicação do teste do Qui-Quadrado verificou-se que houve associação entre o domínio Afetividade-Consistência e as variáveis presença de sequelas e comprometimento das atividades básicas de vida diária, demonstrando que as famílias dos idosos avaliados não estão preparadas para atender ao idoso que necessita de cuidados de forma próxima, afetiva e com regras consistentes.

A dependência de um familiar idoso gera impacto na dinâmica, na economia familiar e na saúde dos membros da família que se ocupam dos cuidados. A evolução dessa dependência pode modificar-se ou até ser preservada, se houver ambiente e assistência adequados. ${ }^{14}$

Enquanto que no domínio Adaptação-Familiar houve interferência apenas da variável nível de escolaridade não-alfabetizados, evidenciando-se que a baixa escolaridade encontrada nos idosos do presente estudo dificulta o desenvolvimento de uma relação baseada em afetos positivos entre os idosos e suas famílias.

O domínio Autonomia apresentou diferença estatística significativa com as variáveis estado civil casado, realização de tratamento e comprometimento das atividades básicas de vida diária, verificando-se desta forma que os idosos avaliados apresentam comprometimento de sua autonomia, privacidade e liberdade nas relações familiares.

\section{CONCLUSÕES}

Diante do exposto, fica evidenciado que a maioria dos idosos entrevistados apresentou comprometimento nos domínios do Inventário de Percepção do Suporte Familiar, o que demonstra, em parte, que estes idosos consideram o seu suporte familiar ruim. Assim, é importante que opções assistenciais sejam oferecidas às famílias com o objetivo de adequar a relação familiar destes idosos.

A família, importante em qualquer fase da vida, torna-se imprescindível na terceira idade, sobretudo em idosos classificados como dependentes que necessitam de cuidados para a realização de suas atividades cotidianas. No entanto, esses cuidados prestados aos idosos dependentes precisam ser qualificados para que possam contribuir com a melhoria da saúde e qualidade de vida destes.
Desta forma, sugere-se que novos estudos sejam realizados no intuito de verificar a relação entre a qualidade dos cuidados prestados aos idosos dependentes pelos seus familiares e a qualidade de vida destes idosos, para que, desta forma, possa ser preconizado por meio de políticas públicas a capacitação da família no cuidado ao idoso.

Entre as limitações deste estudo, pode-se apontar o próprio delineamento da pesquisa, na qual o fato de ser um estudo transversal, impede assegurar relações implícitas de causalidade entre as variáveis estudadas. No entanto, através de fundamentação encontrada na literatura, no que diz respeito ao grau de associação e das relações causais entre variáveis, permite inferir que os resultados do presente estudo parecem suportar as evidências dos modelos explicativos de geração das limitações funcionais na população idosa. Nesta perspectiva sugere-se que novos estudos sejam realizados, levando-se em consideração a divisão dos idosos em grupo, por sexo.

\section{REFERENNCIAS}

1. Silva L, Galera AF, Moreno V. Encontrando-se em casa: uma proposta de atendimento domiciliar para famílias de idosos dependentes. Acta Paul Enferm. 2007 Out-Dez; 20(4):30-9.

2. Souza RF, Skubs T, Bretãs ACP. Envelhecimento e família: uma nova perspectiva para o cuidado de enfermagem. Rev Bras Enferm. 2007 Mai-Jun; 60(3):64-8.

3. Silva MJ, Bessa MEP, Oliveira MC. Tamanho e estrutura familiar de idosos residentes em áreas periféricas de uma metrópole. Cien Enferm. 2004 Jun; 10(1):31-9.

4. Lemos N, Medeiros SL. Suporte social ao idoso dependente. In: Freitas EV, Py L, Neri AL, Caçado FAX, Gorzoni ML, Rocha AM. Tratado de geriatria e gerontologia. Rio de Janeiro (RJ): Guanabara Koogan; 2002. p. 892-8.

5. Pavarini SCI, Luchesi BM, Fernandes HCL, Mendiondo MSZ, Filizola CLA, Barham EJ, et. al. Genograma: avaliando a estrutura familiar de idosos de uma unidade de saúde da família. Rev Eletr Enf. 2008 [acesso 2009 Mar 03]; 10(1):39-50. Disponível em: www.fen.ufg.br/revista/v10/n1/ pdf/v10n1a04.pdf.

6. Tavares DMS, Pereira GA, Iwamoto HH, Miranzzi SSC; Rodrigues LR, Machado ARM. Incapacidade funcional entre idosos residentes em um município do interior de Minas Gerais. Texto Contexto Enferm. 2007 Jan-Mar; 16(1):32-9.

7. Montezuma CA, Freitas MC, Monteiro ARM. A família e o cuidado ao idoso dependente: estudo de caso. Rev Eletr Enf. 2008 [acesso 2009 Mar 03]; 
10(2):395-404. Disponível em: www.fen.ufg.br/ revista/v10/n2/pdf/v10n2a11.pdf.

8. Silveira TM, Caldas CP, Carneiro TF. Cuidando de idosos altamente dependentes na comunidade: um estudo sobre cuidadores familiares principais. Cad Saúde Pública 2006 Ago; 22(8):213-20.

9. Reis LA, Torres, GV, Reis LA. Pain characterization in institutionalized elderly patients. Arq. NeuroPsiquiatr. 2008 Jun; 66(2b):331-5.

10. Reis LA, Mascarenhas CHM, Torres GV. Evaluation of functional capacity on institutionalized elderly in the City of Jequié/BA. Fiep Bulletin 2008; 78(1):8992.

11. Baptista MN. Desenvolvimento do Inventário de Percepção de Suporte Familiar (IPSF): estudos psicométricos preliminares. Psico-USF. 2005 Jan-Jun; 10(1):11-9.
12. Baptista ASD, Neves ATV, Baptista MN. Correlação entre suporte familiar, saúde mental e crenças irracionais em idosos religiosos. Rev Psicol. 2008 Jul-Dez; 9(2):155-64.

13. Luzardo A, Walkman BF. Atenção ao familiar cuidador do idoso com doença de Alzheimer. Acta sci. Health sci. 2004; 26(1):135-45.

14. Herédia VBM, Casara MB, Cortelletti IA. Impactos da longevidade na família multigeracional. Rev Bras Geriatr Gerontol. 2007; 10(1):07-28.

15. Pavarini SCI, Tonon FL, Silva JMC, Mendiondo M, Barham EJ, Filizola CLA. Quem irá empurrar minha cadeira de rodas? A escolha docuidador familiar do idoso. Rev Eletr Enf 2006 [acesso 2009 Mar 03]; 8(3):326-35. Disponível em: http:/ / www.fen.ufg.br/ revista/revista8_3/pdf/v8n3a03.pdf. 